Skinner, F. A. (1951). J. gen. Microbiol. 5, 159-166.

\title{
A Method for Distinguishing Between Viable Spores and Mycelial Fragments of Actinomycetes in Soils
}

\author{
By F. A. SKINNER \\ Soil Microbiology Department, Rothamsted Experimental Station, Harpenden, \\ Hertfordshire
}

SUMMARY: When shaken in suspension, vegetative actinomycete mycelium breaks into a large number of viable fragments which are killed when shaking is prolonged. Spores do not break up into smaller particles and are more resistant to shaking than vegetative fragments. Suspensions of vegetative mycelium may be distinguished from spores by the manner in which the viable count varies with time of shaking. While it is not possible to estimate the numbers of spores and of vegetative particles in mixed suspension the predominating form may be identified. Soil samples from Broadbalk field had a high count of actinomycetes which were almost certainly present as free spores.

Estimates of microbiological activity in soils are usually made by determining the numbers of micro-organisms present by the plating technique. In general, this method is satisfactory for counting unicellular organisms but it cannot be used to give an accurate estimate of the extent of actinomycete (Streptomyces spp.) growth. Since a colony may originate either from a spore or from a fragment of mycelium it is therefore clear that the numbers of colonies appearing on plates give no information about the activity of these organisms at the time of sampling (Waksman, 1927).

Brierley, Jewson \& Brierley (1927) showed that in shaken soil suspensions the viable count of actinomycetes increased steadily with the shaking time. This result may have been caused either by a gradual separation of particles from disintegrating soil crumbs or by a progressive breaking-up of mycelium. Tests in this laboratory have shown the latter explanation to be more probable. A possibility to be considered is that a gradually rising count might not be obtained if the actinomycetes were present in the soil only as spores at the time of sampling. The following experiments were made to study the effect of shaking on suspensions of vegetative mycelium and of spores and to examine the possibility of distinguishing by these means between these two kinds of viable particles.

\section{MATERIALS AND METHODS}

Organism used. All experiments other than those on soil suspensions were made with a pure culture of a single species isolated from Broadbalk soil. It was related to Streptomyces albidoflavus (Rossi Doria) comb. nov.

Preparations of suspensions. Suspensions of vegetative mycelium were prepared by growing the actinomycete in the bottles in which they would eventually be shaken, i.e. a whole culture was shaken for each experiment. Ten $\mathrm{ml}$. portions of nutrient broth ( $\mathrm{NaCl}, 5 \cdot 0 \mathrm{~g}$; Hopkin \& William's peptone, $10.0 \mathrm{~g}$; ; Lab-Lemco, 3.0 g.; Marmite, $1.0 \mathrm{~g}$.; distilled water, 1 1.; adjusted to 
pH 7.2) were added to $1 \mathrm{oz} .(28 \mathrm{ml}$.) culture bottles with sand to assist in breaking up the mycelium. Bottles were then plugged and autoclaved for $15 \mathrm{~min}$. at $15 \mathrm{lb} . / \mathrm{sq}$.in. Each sterile bottle received one drop of a heavy spore suspension and was incubated at $25^{\circ}$. Cultures prepared in this way showed good bottom vegetative growth consisting of a large number of minute colonies, in a few days. Before shaking, the plug was replaced by a sterile metal screwcap and the bottle agitated gently by hand for $2 \mathrm{~min}$. to render the suspension reasonably homogeneous. An initial sample to give the count at zero time was then taken. Shaking was performed by a reciprocating machine in which the bottles moved through a vertical distance of $9 \mathrm{~cm}$. at a rate of about 265 strokes/min. Samples $(0.5 \mathrm{ml}$.) were taken from the bottles at intervals during the experiment, for dilution in sterile saline $(0.75 \%(\mathrm{w} / \mathrm{v}) \mathrm{NaCl})$ and plating. Counts were made on three replicate plates for each sample after 7 days' incubation.

Spore suspensions were made by washing the sporulating surfaces of 10-day-old slope cultures of the actinomycete on Waksman's starch-tryptone agar, with a detergent consisting of a $0.1 \%(\mathrm{v} / \mathrm{v})$ solution of Calsolene Oil HS (I.C.I. (Pharmaceuticals) Ltd.) in distilled water. Washings were bulked and $10 \mathrm{ml}$. portions transferred to empty sterile culture bottles. These suspensions were shaken and sampled in the same way as those of vegetative mycelium.

\section{EXPERIMENTAL}

As a preliminary experiment (Exp. 1), $250 \mathrm{ml}$. of a suspension of vegetative mycelium were shaken by hand in a Roux bottle with 25 g. sand. The viable count increased with time of shaking; rapidly at first and then more slowly, indicating the progressive fragmentation of pieces of mycelium. This result was similar to those obtained by Brierley et al. for soil suspensions. Their results could thus be explained by supposing the actinomycetes to be present in these soils in an active vegetative state. It should be noted, however, that the gradual rise in count in the above experiment occurred in the presence of a considerable quantity of sand, whereas in the experiments of Brierley et al. no sand was present other than that in the soil samples. Further experiments were made to determine whether vegetative mycelium would be broken up in this way when suspensions were shaken without abrasive. Mechanical shaking was employed to standardize technique and to make possible the treatment of several suspensions at the same time.

\section{Mechanical shaking of vegetative mycelium}

Exp. 2. Four 9-day-old vegetative cultures were prepared, the bottles containing sand in the quantities shown: A, no sand; B, 1 g. sand; C, 2 g. sand; $\mathrm{D}, 5 \mathrm{~g}$. sand. These cultures were shaken for $1 \mathrm{hr}$. and sampled as previously described. The result for culture A showed that the shaking of mycelium without sand could cause a gradual breaking-up of the fragments (Fig. 1). A similar increase in the number of viable particles was found for $\mathbf{B}$ and $\mathbf{C}$. It was evident that though sand assisted the fragmentation of mycelium considerably, 
its presence was not essential for the production of a gradually rising colony count of the type found by previous workers (Brierley et al., 1927) for soil suspensions. Clearly, their results were consistent with the view that actinomycetes were existing in a vegetative state in those soils examined. A very different result was obtained for culture $\mathbf{D}$. The colony count increased rapidly at first, to reach its highest value at the $15 \mathrm{~min}$. sampling time. Numbers then fell sharply as shaking was continued, demonstrating rapid killing of the fragments. Curran \& Evans (1942) found that bacterial spores were killed when shaken in suspension with inert particles, killing being evident from the beginning of the experiment. A similar result for vegetative cells was found by King \& Alexander (1948). It was probable, therefore, that some fragments of mycelium were killed from the beginning in all suspensions containing sand but that the rate of killing was masked by the more rapid formation of new fragments from the larger pieces of mycelium. After shaking for $15 \mathrm{~min}$. it was clear that the rate of killing exceeded the rate of formation of new particles.

The result for culture $\mathbf{D}$ was confirmed by an additional experiment (Exp. 3) in which a $10 \mathrm{ml}$. vegetative culture was shaken with $5 \mathrm{~g}$. sand. A larger number of samples (each of $0.1 \mathrm{ml}$.) were taken over a shaking period of $90 \mathrm{~min}$., to provide adequate data on the decline in numbers. The points obtained by plotting logarithms of the colony counts for samples taken after the $15 \mathrm{~min}$. sampling time, against the shaking time, lay about a straight line. This showed that the resultant of the rates of killing and of formation of new fragments was an exponential decline. Both Curran \& Evans (1942) and King \& Alexander (1948) observed that bacteria were killed exponentially when shaken in suspension with glass beads. Since there is no evidence to suggest that small pieces of mycelium will be destroyed according to some different mathematical law it may be inferred that there was practically no formation of new fragments after shaking for $15 \mathrm{~min}$. Thus, from these experiments, it appears that the initial effect of shaking on suspensions containing vegetative growth is to break up the pieces of mycelium with a consequent increase in the number of viable particles. Prolonged shaking in the presence of sand brings about a progressive killing of these fragments.

\section{Mechanical shaking of spore suspensions}

Exp. 4. Two $10 \mathrm{ml}$. portions of spore suspension (A, no sand; B, with $5 \mathrm{~g}$. sand) were shaken as described. Counts for both suspensions were initially high and remained fairly constant during the whole shaking period (Table 1). There was no evidence that the spores could break up into smaller viable particles or that prolonged shaking even with $5 \mathrm{~g}$. sand had much effect on their viability (cf. Exps. 2 and 3).

These differences between the results for spore suspensions and those for vegetative suspensions were very marked. In the former case, there was little variation among colony counts for different times of shaking, at least during the first $15 \mathrm{~min}$. of the experiments. Whenever the actinomycete was present in the mycelial condition, however, then evidence of fragmentation of that mycelium was given by a rising count over the same shaking period. A con- 
Table 1. Suspensions of actinomycete spores shaken mechanically

(Dilution: 1/20,000.)

\begin{tabular}{|c|c|c|}
\hline \multirow{2}{*}{$\begin{array}{l}\text { Time of shaking } \\
\text { (min.) }\end{array}$} & \multicolumn{2}{|c|}{$\begin{array}{c}\text { Colonies/plate } \\
\text { (mean of three replicate plates) }\end{array}$} \\
\hline & $A$ (no sand) & B (5 g. sand) \\
\hline 0 & $443 \cdot 3$ & $328 \cdot 0$ \\
\hline $\mathbf{5}$ & $322 \cdot 0$ & $468 \cdot 0$ \\
\hline 15 & $296 \cdot 0$ & $448 \cdot 7$ \\
\hline 30 & $319 \cdot 3$ & $412 \cdot 7$ \\
\hline 60 & $296 \cdot 7$ & $371 \cdot 3$ \\
\hline
\end{tabular}

venient way of representing such results without regard for the actual numbers of viable spores or fragments in any experiment, is to take the ratio of the count at some particular time (e.g. $15 \mathrm{~min}$.) to the initial count. This ratio is large for suspensions of vegetative mycelium but approximates to unity for suspensions of detached spores. Ratios derived from all the experiments are given in Table 2.

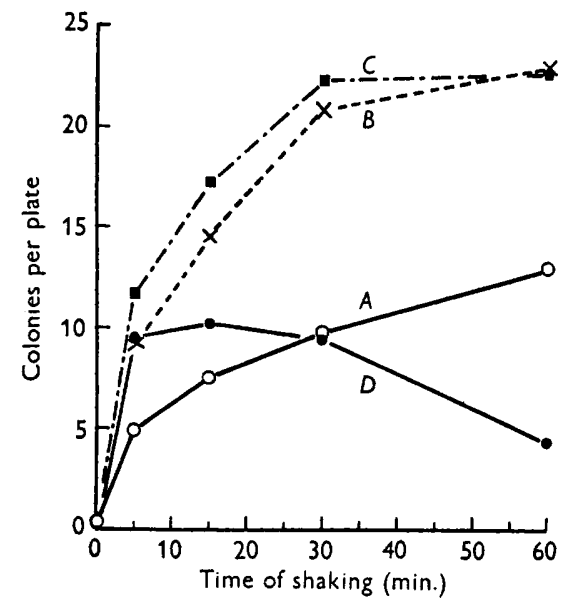

Fig. 1

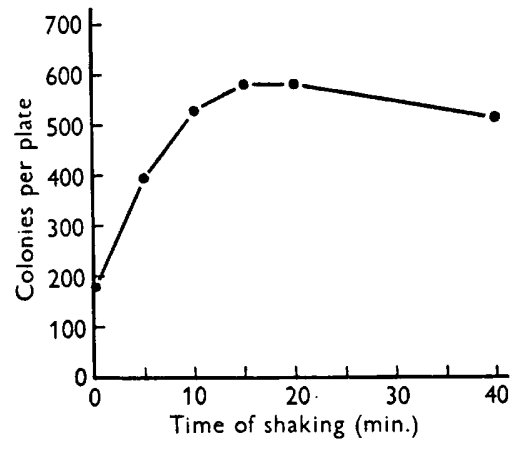

Fig. 2

Fig. 1. Suspensions of pieces of vegetative actinomycete mycelium shaken mechanically (Exp. 2). A, without sand; B, with 1 g. sand; C, with 2 g. sand; D, with 5 g. sand. Plating dilution: $1 / 20,000$.

Fig. 2. Suspension of actinomycete soil culture shaken mechanically with $5 \mathrm{~g}$. of sand (Exp. 10). Plating dilution: 1/250,000.

\section{Suspensions of soil}

In view of these results it seemed probable that information about the state in which actinomycetes were present in soil might be obtained by shaking soil suspensions in the manner described, and observing the way in which the count of Streptomyces colonies varied with the shaking time.

Experiments were made with soil samples taken from the following three plots of Broadbalk field (permanent wheat): plot 2, receiving 14 tons of dung/ 
acre/year; plot 3, untreated; plot 7, receiving complete minerals (3.5 cwt. superphosphate, 2 cwt. potassium sulphate, 1 cwt. sodium sulphate, 1 cwt. magnesium sulphate and $4 \mathrm{cwt}$. ammonium sulphate/acre/year).

Table 2. Ratios between colony counts at 15 min. sampling times and initial counts

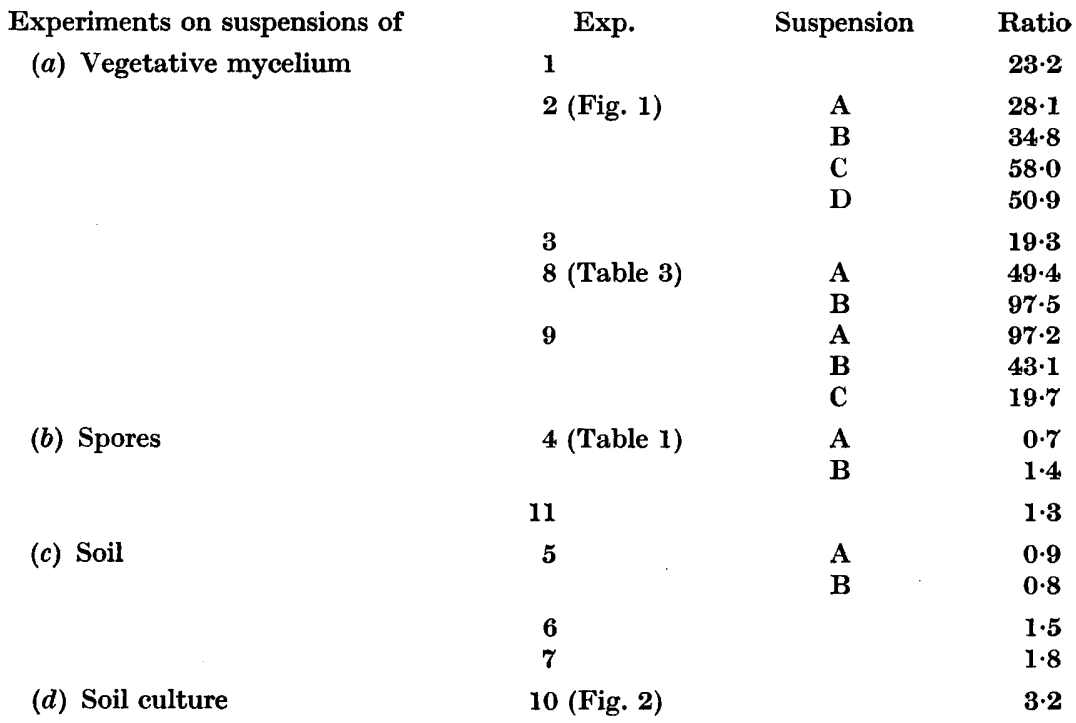

Exp. 5. Five g. of sieved plot 2 soil were added to $10 \mathrm{ml}$. sterile saline (suspension A): a duplicate suspension (B) contained in addition 5 g. sand. These were shaken and sampled in the usual way. Plates were examined microscopically to distinguish Streptomyces colonies from those of other organisms. A high initial count of Streptomyces spp. was found for each suspension. These counts varied little during the experiment, though a slight fall occurred between the 20 and $40 \mathrm{~min}$. sampling times in the suspension shaken with sand. It was therefore considered probable that in this soil actinomycete spores were present in abundance but that there was little if any vegetative mycelium. Similar results were found for soils taken from plots 3 (Exp. 6) and 7 (Exp. 7).

\section{Detection of small quantities of vegetative mycelium in suspension}

The experiments on pure cultures of actinomycete mycelium (Exps. 2 and 3) were made with suspensions containing considerable amounts of vegetative growth (c. $10 \mathrm{mg}$. dry wt./10 ml. culture). However, it is unlikely that such a large quantity of mycelium would ever be found in a suspension of natural soil of the concentration used in Exps. 5-7. It was therefore considered necessary to determine whether mycelial fragments in very low concentration would be broken up sufficiently quickly on shaking to give the rapid initial rise in numbers so characteristic of heavier suspensions. 
Exp. 8. Samples $(\mathbf{1} \cdot 0 \mathrm{ml}$.) of a $\mathbf{2}$-day-old broth culture of vegetative mycelium were diluted $1 / 10$ and $1 / 100$ in saline. In the latter suspension only a very few minute flecks of mycelial growth could be seen. Ten ml. portions of both suspensions were shaken with $5 \mathrm{~g}$. quantities of sand. The results (Table 3) showed the same rise and fall in numbers as was previously found for heavier mycelial suspensions shaken with this quantity of sand; so it was concluded that mycelium could be detected by this method even when present in very small quantities.

Table 3. Suspensions of vegetative actinomycete mycelium shaken mechanically

\begin{tabular}{|c|c|c|}
\hline \multirow{2}{*}{$\begin{array}{l}\text { Time of shaking } \\
\text { (min.) }\end{array}$} & \multicolumn{2}{|c|}{$\begin{array}{c}\text { Colonies/plate } \\
\text { (mean of three replicate plates) } \\
\text { Vegetative culture diluted: }\end{array}$} \\
\hline & A, $1 / 10$ in saline & $\mathrm{B}, 1 / 100$ in saline \\
\hline 0 & $2 \cdot 7$ & $1 \cdot 3$ \\
\hline 5 & $151 \cdot 0$ & $180 \cdot 0$ \\
\hline 15 & $133 \cdot 3$ & $126 \cdot 7$ \\
\hline $\mathbf{3 0}$ & $69 \cdot 7$ & $37 \cdot 0$ \\
\hline 60 & $12 \cdot 7$ & $\mathbf{3} \cdot \mathbf{3}$ \\
\hline Plating dilutions & $1 / 2000$ & $1 / 200$ \\
\hline
\end{tabular}

The effect of soil particles on the fragmentation of mycelium

No evidence was found for the presence of mycelium in suspensions of natural soils. The suspensions used, however, contained large amounts of soil $(5 \mathrm{~g} . /$ $10 \mathrm{ml}$.) and such a high concentration of soil particles might prevent the breaking of any mycelial fragments present, thereby giving a result apparently indicating the absence of mycelium. The following experiment (Exp.9) was made to determine the effect of different amounts of soil on the rate of break-up of vegetative fragments. Three suspensions, each consisting of $10 \mathrm{ml}$. of a 1/100 dilution of vegetative culture in saline, were prepared. Sterile soil was then added to the bottles in the following quantities: A, no soil; $\mathrm{B}, 0.5 \mathrm{~g}$. soil; C, 5.0 g. soil: each bottle also contained $5 \mathrm{~g}$. sand. Shaking and sampling were performed in the usual way. The results were characteristic of those always found for vegetative suspensions, though it was clear that a high concentration of soil particles (as in C) diminished the maximum count considerably either by preventing fragmentation through restricting movement of the sand grains, or by increasing the death-rate. It was concluded that in spite of the presence of a large amount of soil in suspension, very small quantities of mycelium in that suspension could still be detected by the shaking technique.

\section{The effect of shaking a soil culture of Streptomyces sp. containing both spores and vegetative mycelium}

It has been shown that suspensions of vegetative actinomycete mycelium can be distinguished from those of spores. It has also been shown that actinomycetes were probably present as spores in the soils examined. In some soils, 
however, it is to be expected that both spores and vegetative mycelium will be present together, in which case shaken suspensions of these soils should give results different from those found for Broadbalk soils. An experiment was made on a pure soil culture known to contain both vegetative mycelium and spores of the actinomycete to determine whether these two kinds of viable particles could be distinguished when present in the same suspension.

A soil culture was prepared as follows: $30 \mathrm{~g}$. of sieved, air-dried Broadbalk soil (plot 3) were placed in a Petri dish and autoclaved for $1 \mathrm{hr}$. A suspension of spores in $12 \mathrm{ml}$. nutrient broth was distributed over the soil surface. After incubation for 7 days the actinomycete had made good growth, aerial mycelium being visible on many of the soil crumbs. Five g. of this soil culture were added to $10 \mathrm{ml}$. saline in a shaking bottle with $5 \mathrm{~g}$. sand and treated in the usual way (Exp. 10). The results (Fig. 2) yielded a curve which showed some features characteristic of curves derived both from spore and mycelial suspensions. The high initial count was almost certainly caused by a rapid release of spores from the soil particles, and the rise in count during the first $15 \mathrm{~min}$. of shaking indicated the break-up of vegetative mycelium. It was considered possible, however, that this rise in count might have been caused partly by a gradual breaking-up of spore aggregates and sporogenous aerial hyphae. To determine the rate at which such aggregates would be disrupted on shaking a further experiment was made. The aerial growth was removed from an agar slope culture of the actinomycete by dragging a wire loop across the surface, and added to $10 \mathrm{ml}$. saline with $5 \mathrm{~g}$. sand in a shaking bottle (Exp. 11). On shaking, a high initial count was obtained. This was followed by a slight rise in count after shaking for $5 \mathrm{~min}$. and then by a fall. The occurrence of the highest count so early in the experiment showed that spore aggregates were broken up very easily. The subsequent decline in numbers was not so readily explained, but probably it resulted from the destruction of undifferentiated aerial hyphae which formed part of the surface growth removed by the wire loop. In view of these results it was thought probable that the rising count obtained in the soil culture experiment was due almost entirely to the breaking-up of mycelial fragments. It seemed clear from these results that the shaking technique described could be used to analyse mixed suspensions of spores and vegetative fragments.

\section{DISCUSSION}

The evidence presented suggests that species of Streptomyces were present in Broadbalk soils as spores at the time of sampling. A similar conclusion for Indian soils was reached by Subrahmanyan (1929) who claimed to be able to distinguish between colonies of conidial and mycelial origin. Whether actinomycetes are usually present in this dormant state in soils has not yet been established, but it is evident that vegetative development must sometimes occur even though such periods of activity may be of short duration. Results (Exp. 9) have shown that vegetative growth could be detected in soil culture by the use of shaken suspensions. Support has also been given (Exp. 1 and 2) to the opinion that vegetative mycelium was present in soils examined by 
other workers (Brierley et al. 1927). Other methods such as the direct observation of glass slides which have been buried in soil may be used to study the development of actinomycetes in soil culture (Jensen, 1943), but it is an advantage to be able to detect the presence of vegetative mycelium and spores at any instant of time in natural soils. The shaking technique described may be used to obtain such information and so assist in the solution of a complex problem. It is clear, however, that any attempt to make these methods useful for quantitative estimations should be delayed until further investigations have been made on the behaviour of other species of Streptomyces in shaken suspensions.

I wish to express my thanks to the Agricultural Research Council for the grant which made this work possible, to Dr H. G. Thornton, F.R.S., and Dr Jane Meiklejohn for helpful advice and criticism, and to Miss Mabel Dunkley for assistance in preparing the manuscript.

\section{REFERENCES}

Brierley, W. B., Jewson, S. F. \& Brierley, M. F. (1927). The quantitative study of soil fungi. Proc. First Int. Congr. Soil Sci., Wash.; Commission III, p. 48.

Curran, H. R. \& Evans, F. R. (1942). The killing of bacterial spores in fluids by agitation with small inert particles. J. Bact. 43, 125.

Jensen, H. L. (1943). Observations on the vegetative growth of actinomycetes in the soil. Proc. Linn. Soc. N.S.W. 68, 67.

KING, H. K. \& Alexander, H. (1948). The mechanical destruction of bacteria. J. gen. Microbiol. 2, 315.

Subrammanyan, V. (1929). Studies on soil actinomyces. II. Their mode of occurrence in the soil. J. Indian Inst. Sci. 12, (A) 57.

Waksman, S. A. (1927). Principles of Soil Microbiology, 1st ed. London: Baillière, Tindall and Cox.

(Received 15 April 1950) 